\title{
Comparative studies of electrospinning and solution blow spinning processes for the production of nanofibrous poly(L-lactic acid) materials for biomedi- cal engineering
}

\author{
Michal Wojasiński*, Maciej Pilarek, Tomasz Ciach \\ Warsaw University of Technology, Faculty of Chemical and Process Engineering, Biotechnology and Bioprocess Engineering \\ Division, BioMedical Engineering Laboratory, Waryńskiego 1, 00-645 Warszawa, Poland \\ "Corresponding author: e-mail: m.wojasinski@ichip.pw.edu.pl
}

\begin{abstract}
Comparative statistical analysis of the influence of processing parameters, for electrospinning (ES) and solution blow spinning (SBS) processes, on nanofibrous poly(L-lactic acid) (PLLA) material morphology and average fiber diameter was conducted in order to identify the key processing parameter for tailoring the product properties. Further, a comparative preliminary biocompatibility evaluation was performed. Based on Design of Experiment (DOE) principles, analysis of standard effects of voltage, air pressure, solution feed rate and concentration, on nanofibers average diameter was performed with the Pareto's charts and the best fitted surface charts. Nanofibers were analyzed by scanning electron microscopy (SEM). The preliminary biocompatibility comparative tests were performed based on SEM microphotographs of CP5 cells cultured on materials derived from ES and SBS. Polymer solution concentration was identified as the key parameter influencing morphology and dimensions of nanofibrous mat produced from both techniques. In both cases, when polymer concentration increases the average fiber diameter increase. The preliminary biocompatibility test suggests that nanofibers produced by ES as well as SBS are suitable as the biomedical engineering scaffold material.
\end{abstract}

Keywords: electrospinning, solution blow spinning, nanofibers, biomedical engineering, design of experiments, biocompatibility.

\section{INTRODUCTION}

In the last years, polymeric nanofibrous materials have gained a continuously growing scientific, technological and industrial significance. Known since 1930 - patent of A. Formhals ${ }^{1}$ on nanofibers manufacturing method, materials composed from nanofibers found application spreading in different fields such as air and water filtration $^{2}$, cosmetics, textile industry ${ }^{3}$, active materials for photonics and electronics ${ }^{4}$, and gathering more and more attention in biomedical fields like wound dressing ${ }^{5}$, controlled drug delivery ${ }^{6}$, and most of all tissue engineering and regenerative medicine ${ }^{7-9}$. Interesting properties of nanofibrous materials including high surface to volume ratio, high and tunable porosity, biocompatibility (depended on applied polymer) and easy post processing (Fig. 1.) make these materials highly desirable.

Several fabrication and synthesis methods are currently available, most of them applied at laboratory scale, for the production of polymeric nanofibers, such as template synthesis, phase separation, self assembly ${ }^{\mathbf{1 0}}$, solvent casting, and solid freeform fabrication technique ${ }^{11}$. However, the electrospinning, also known as electrostatic spinning (ES), is a technology, which, because of the simplicity and product properties, is the most widely used for nanofibers preparation in laboratory scale. Since 1990, the number of scientific reports concerning electrospinning has grown from a few articles per year to almost half of thousand in $2011^{12,13}$. Still, despite the intense research on electrospinning, there are only few reports about the application of this technique for the industrial production of nanofibers ${ }^{13}$. Low efficiency of ES technique, which allows using milliliters of polymer solution during the fibers formation process, makes ES a method in need of improvement or replacement. Possible modifications of ES setup allowing improvements of process efficiency include multi-jet electrospinning ${ }^{14}$ or needleless electrospinning ${ }^{15}$. However there are still basic problems with jet interactions or maintaining a thin film of polymer solution in the aforementioned methods which must be solved.

Due to the fact that electrospinning is not easy to up-scale, solution blow spinning (SBS) technique for producing nanofibers was recently proposed ${ }^{16}$. Based on the principles of ES and melt-blowing technologies to make nanofibers with diameter range similar to ES method, SBS gives an opportunity to process ten times more polymer solution in the laboratory scale. Solution blow spinning is based on the high speed of decompressed air causing rapid stretching and evaporation of solvent from polymeric solution jet at the outlet of the concentric nozzles system ${ }^{17}$. Application of high-pressured air allows SBS to be scaled-up easily, rather than electrospinning, where the high voltage is applied.

The paper is focused on the nanofibers formation for biomedical purposes, like tissue engineering or regenerative medicine. In our study, as a model polymer for nanofibers formation by electrospinning and solution blow spinning poly(L-lactic acid) (PLLA) was used. FDA approved, biodegradable and biocompatible polyester - PLLA is commonly used in many biomedical applications, e.g. tissue engineering and regenerative medicine, wound dressing, drug delivery and so on $^{18}$. Also, PLLA is one of the most important materials for nanofibers formation, especially for the preparation of nanofibers for bioengineering ${ }^{19,20}$.

The basic aim of this work was to identify key process parameters influencing electrospun and solution blow spun nanofibrous mat's properties, i.e. morphology and average fiber diameter, and comparison of the influence of process parameters on product properties in both 
methods. The preliminary studies of biocompatibility of electrospun and solution blow spun mats were performed. We investigated how the changes of applied voltage, air pressure, solution feed rate, and solution concentration influence the product properties for both methods by applying specific Design of Experiment (DOE). Detailed statistical analysis of the results of the experiments has been performed and the results of the aforementioned analysis were presented in the form of Pareto's charts and best fitted surface charts. Preliminary biocompatibility tests were performed on PLLA-based materials obtained from both techniques by in vitro cell culture of cartilage cells (CP5 chondrocytes).

\section{EXPERIMENTAL}

\section{Polymer solutions}

PLLA with weight-average molecular weight about 200 $\mathrm{kDa}$ (Polysciences, Inc., USA) was used for preparing the solutions for electrospinning and solution blow spinning processes. Mixture of dichloromethane and N,N-dimethylformamide $(9: 1, \mathrm{v} / \mathrm{v})$ was used as a solvent for PLLA solution for electrospinning, and mixture of chloroform and acetone $(3: 1, \mathrm{v} / \mathrm{v})$ was used as a solvent mixture for PLLA solubilisation prior solution blow spinning. Solvents used in experiments were analytical grade purchased from Carlo Erba Reagents, Italy. Concentrations of individually tested solutions are listed in Table 1 . Solutions for both processes were prepared 24 hours before the experiments. Preparation of solutions was conducted in ambient conditions, with relative humidity $(\mathrm{RH})$ varied from about 40 to $55 \%$.

Table 1. Fractional plan of experiments $\left(3^{\mathrm{k}-\mathrm{P}}\right)-$ combination of values for the experiments

\begin{tabular}{|c|c|c|c|c|}
\hline $\begin{array}{l}\text { Voltage } \\
{[\mathrm{kV}]}\end{array}$ & $\begin{array}{c}\text { Air pressure } \\
{[\mathrm{kPa}]}\end{array}$ & $\begin{array}{r}\text { Solu } \\
{[\mathrm{n}}\end{array}$ & $\begin{array}{l}\text { feed } \\
\left.h^{-1}\right]\end{array}$ & $\begin{array}{c}\text { Concentration } \\
{[\% \mathrm{w} / \mathrm{w}]}\end{array}$ \\
\hline ES & SBS & ES & SBS & ES \& SBS \\
\hline 12 & 500 & 1 & 15 & 4 \\
\hline 12 & 500 & 2 & 30 & 4 \\
\hline 12 & 500 & 3 & 45 & 4 \\
\hline 15 & 1000 & 1 & 15 & 4 \\
\hline 15 & 1000 & 2 & 30 & 4 \\
\hline 15 & 1000 & 3 & 45 & 4 \\
\hline 18 & 1500 & 1 & 15 & 4 \\
\hline 18 & 1500 & 2 & 30 & 4 \\
\hline 18 & 1500 & 3 & 45 & 4 \\
\hline 12 & 500 & 1 & 15 & 6 \\
\hline 12 & 500 & 2 & 30 & 6 \\
\hline 12 & 500 & 3 & 45 & 6 \\
\hline 15 & 1000 & 1 & 15 & 6 \\
\hline 15 & 1000 & 2 & 30 & 6 \\
\hline 15 & 1000 & 3 & 45 & 6 \\
\hline 18 & 1500 & 1 & 15 & 6 \\
\hline 18 & 1500 & 2 & 30 & 6 \\
\hline 18 & 1500 & 3 & 45 & 6 \\
\hline 12 & 500 & 1 & 15 & 8 \\
\hline 12 & 500 & 2 & 30 & 8 \\
\hline 12 & 500 & 3 & 45 & 8 \\
\hline 15 & 1000 & 1 & 15 & 8 \\
\hline 15 & 1000 & 2 & 30 & 8 \\
\hline 15 & 1000 & 3 & 45 & 8 \\
\hline 18 & 1500 & 1 & 15 & 8 \\
\hline 18 & 1500 & 2 & 30 & 8 \\
\hline 18 & 1500 & 3 & 45 & 8 \\
\hline
\end{tabular}

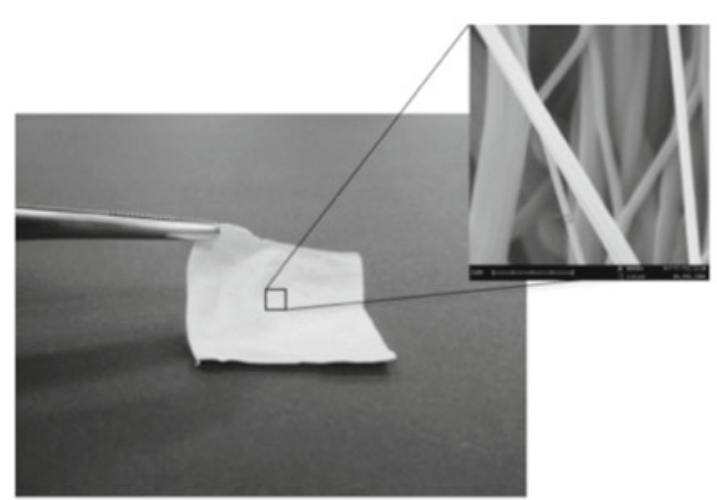

Figure 1. Nanofibrous mat produced by solution blow spinning

\section{Electrospinning}

Electrospun nanofibrous polymeric mats were prepared in the apparatus shown in Figure 2a. Polymer solution was supplied into the spinneret by syringe pump with a controlled feeding rate. The spinneret was connected to the positive electrode of high voltage power supply. Thus, polymer solution was charged and stretched by electrostatic field produced between spinneret and grounded aluminum plate. During the stretching process the solvent evaporates from the polymer solution giving fibers. The plate served as a collector for electrodeposited nanofibrous mats and was placed $120 \mathrm{~mm}$ from the spinneret. ES process for the PLLA fibers production was conducted in ambient conditions, with $\mathrm{RH}$ in the range of $45-50 \%$.

\section{Solution blow spinning}

Solution blow spinning system is shown in Figure $2 \mathrm{~b}$. The main part of the presented apparatus is a system of the concentric nozzles designed specifically for this process. Polymer solution is supplied through the inner

a)

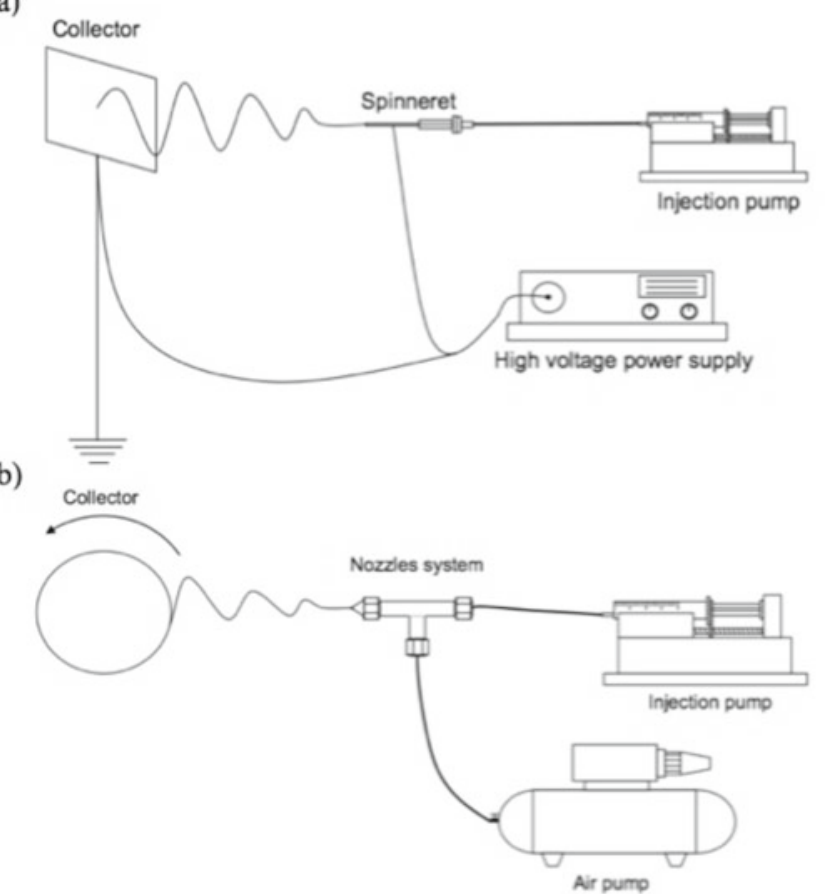

Figure 2. The scheme of: electrospinning setup (a); solution blow spinning setup (b) 
nozzle of nozzles system by syringe pump with controlled feeding rate, while compressed gas, in this case - air, is supplied through the outer nozzle. Based on Bernoulli's principle, due to the rapid decompression of air the velocity of gas rises. Thus, on account of viscous interaction between the gas and the solution of polymer, the conical-shaped structure is formed at the outlet of inner nozzle ${ }^{16}$. Polymer nanofibers were dragged from the cone by viscous forces of flowing air overcoming the surface tension forces. Fine polymer nanofibers were collected on rotating drum placed $200 \mathrm{~mm}$ from the nozzles system. SBS process for the PLLA fibers production was conducted in ambient condition, with $\mathrm{RH}$ in the range of $45-50 \%$.

\section{Viscosity measurements}

The viscosity measurements of polymer solutions used in both electrospinning and solution blow spinning were conducted with a Rotational Viscometer (Fungilab S.A., Spain) at rotational speed of spindle between 6 and 30 rpm. Viscosity measurements were performed in ambient conditions, after preparation of solutions, before the spinning processes.

\section{Statistical analysis}

Research was conducted based on a trivalent fractional plan of experiment $\left(3^{\mathrm{k}-\mathrm{P}}\right)$ generated in Statistica 10 software using Design of Experiments tool. Investigation of significant influence of the three key parameters for each nanofibrous producing methods was conducted, voltage, solution feed rate and solution concentration for ES, air pressure, solution feed rate and solution concentration for SBS. The experiments were performed in three for three levels of each parameter to fit quadratic model. The resulting 27 combinations of values of investigated parameters are listed in Table 1. The main effects and interactions between the investigated parameters were analyzed by preparing Pareto's charts and best fitted surface charts, respectively. The average values of fiber diameter for each experiment were calculated based on the measurement of at least fifty fibers per sample. The results of all experiments are shown as an average diameter of fibers measured for each sample \pm standard deviation of mean.

Pareto chart indicates the influence of all processing parameters i.e. factors on the output value, in this case - average fiber diameter. The most important parameter affecting product properties might be found based on Pareto chart. The length of each bar in the chart represents a standardized effect of the corresponding factor in quadratic (Q) and linear (L) model of the response of the system. The fact that bar corresponding with certain factor is outside of the line of significance (on the right side of the line) indicates that this factor have an influence on investigated quality factor. The negative sign of the influence of the certain factor means decreasing of the quality factor with increasing the value of this factor. The positive sign of the influence means increasing of the quality factor with increasing the value of certain factor. The best-fitted surface charts were prepared in order to compare differences in the dependence of both investigated processes in function of all processing parameters. The character of the changes of quality factor for both processes might be compared based on the best-fitted surface charts.

\section{Microscope analysis}

The size and morphology of materials obtained in electrospinning and solution blow spinning, and morphology of cells cultured on investigated materials were determined based on the analysis of microphotographs from scanning electron microscope (SEM, FEI PHENOM ${ }^{\mathrm{TM}}$, USA). Samples for SEM observations were cut into square pieces $10 \mathrm{~mm} \times 10 \mathrm{~mm}$ and gold-coated (K550 Emitech, USA) prior the analysis. Adobe Photoshop CS6 software measuring tool was used for SEM micrographs analysis. Ten SEM images were analyzed per sample in order to determine differences in morphology. The diameter of fifty single fibers was measured in order to calculate the average fiber diameter for each material obtained in ES and SBS process.

\section{CP5 cell culture}

The CP5 cell line, bovine (Bos taurus, Holstein-Fresian) articular cartilage progenitor cells ${ }^{21}$ was used for biocompatibility test. The CP5 cells have been purchased from European Collection of Cell Cultures (Public Health England, Salisbury, United Kingdom). The CP5 chondrocytes were maintained in Dulbecco's Modified Eagle Medium/Nutrient Mixture F-12 (DMEM/F12) supplemented with GlutaMAX ${ }^{\circledR}, 10 \%$ fetal calf serum (FCS), $50 \mu \mathrm{L} \mathrm{mL}^{-1}$ ascorbate, $10 \mathrm{mM}$ HEPES buffering agent and antibiotics $\left(0.05 \mathrm{u} \mathrm{mL}^{-1}\right.$ penicylin, $0.05 \mathrm{u} \mathrm{mL}^{-1}$ streptomycin) at $37^{\circ} \mathrm{C}$ according to procedure obtained from supplier of cell line ${ }^{21}$. DMEM/F12, FCS and all chemicals were obtained from Invitrogen Co. (USA) and were of animal cell culture quality.

The CP5 adherent cells were cultured in closed and sealed 24 well-plates (Becton-Dickinson, USA) in DMEM/F12. Chondrocytes were cultured (i) on fibrous scaffolds from both, electrospinning and solution blow spinning, placed on the bottom of the well, and (ii) directly on solid surface of polystyrene (well's bottom) in monolayer form as a control culture. In both cases of culture, the chondrocytes were cultured in $1 \mathrm{~mL}$ of DMEM/F12. Inoculum of CP5 cell line was prepared from, i.e. $75-80 \%$, confluent cultures, passaged every 4-5 days. The cells were flushed with PBS (Invitrogen Co., USA), incubated in $0.25 \%$ trypsin (Invitrogen Co., USA) for about $3 \mathrm{~min}$. at $37^{\circ} \mathrm{C}$. The number of the cells was estimated using Malassez hemocytometer (Brand, Germany). The cells were suspended in DMEM/F12 and seeded into the 24 well plates to obtain initial density of $2 \cdot 10^{5}$ cells $\mathrm{mL}^{-1}$.

\section{RESULTS}

\section{Viscosity measurements}

The values of viscosity measured for all solutions are listed below. For PLLA solution in DCM/DMF solvents mixture viscosity for solutions of PLLA concentration $4 \% \mathrm{w} / \mathrm{w}, 6 \% \mathrm{w} / \mathrm{w}$ and $8 \%$ was respectively $24.79 \pm 0.06$ $\mathrm{mPa} \cdot \mathrm{s}, 44.65 \pm 0.06 \mathrm{mPa} \cdot \mathrm{s}, 142.25 \pm 8.88 \mathrm{mPa} \cdot \mathrm{s}$. For PLLA solution in chloroform/acetone solvents mixture viscosity for solutions of PLLA concentration $4 \% \mathrm{w} / \mathrm{w}$, 
$6 \% \mathrm{w} / \mathrm{w}$ and $8 \% \mathrm{w} / \mathrm{w}$ respectively $32.72 \pm 0.05 \mathrm{mPa} \cdot \mathrm{s}$, $125.38 \pm 4.39 \mathrm{mPa} \cdot \mathrm{s}, 407.11 \pm 23.62 \mathrm{mPa} \cdot \mathrm{s}$.

\section{Statistical analysis}

It is well established in literature that ES process highly depends on processing parameters, i.a. solution concentration and viscosity, voltage and solution feed rate ${ }^{22}$, and SBS process depends on parameters like solution concentration and viscosity, gas pressure and solution feed rate ${ }^{\mathbf{1 7}}$. Significance of all processing parameters, for both investigated methods, was identified by preparing Pareto chart for standardized effect evaluation (Fig. 3).

The most significant factor for both, electrospinning and solution blow spinning is the concentration of polymer solution used in the process of preparation of

a)

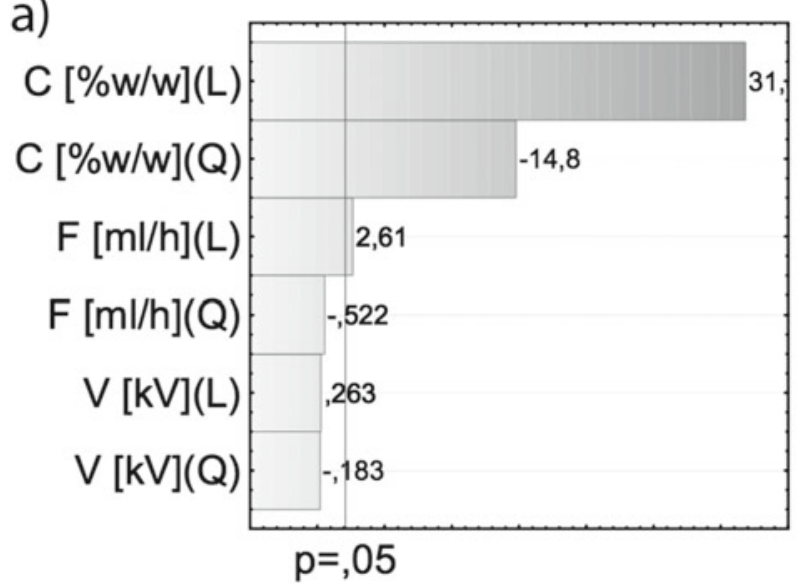

nanofibrous material (Fig. 3). It is well established for the ES process that changing polymer concentration in the solution keeping other parameters fixed causes drastic changes in fibers morphology ${ }^{23}$. As well as ES process, in SBS process dependence of fibers morphology in function of polymer concentration in the solution was reported ${ }^{24}$. The results of analysis of simultaneous changes of processing parameters for ES and SBS process indicates that average fiber diameter highly depends on the changes of polymer solution concentration, in case of PLLA solutions, and it increases with increasing the polymer concentration in the solution (Fig. 4). It is caused by solution viscosity, which also increases with concentration of polymer in the solution. This phenomenon is the same for both processes.

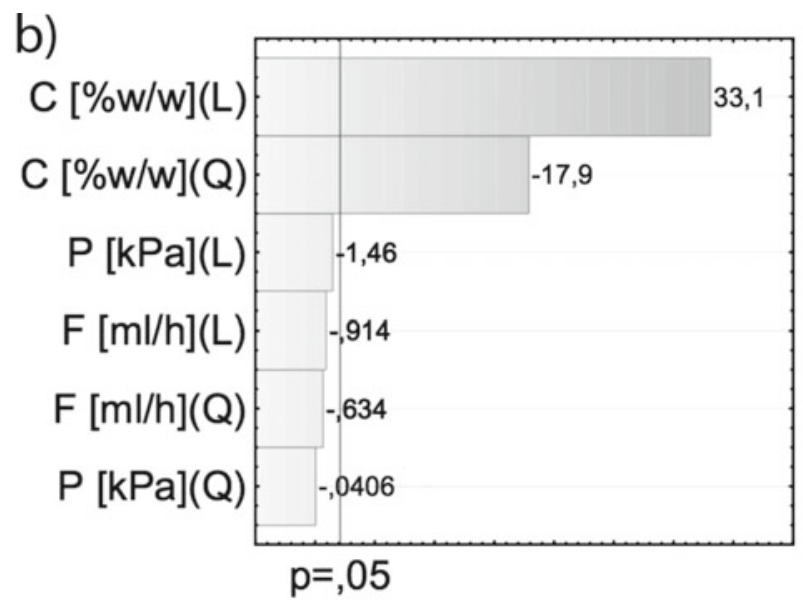

Figure 3. Standard effect estimation as Pareto chart of: electrospinning process (a); solution blow spinning process (b)

a)

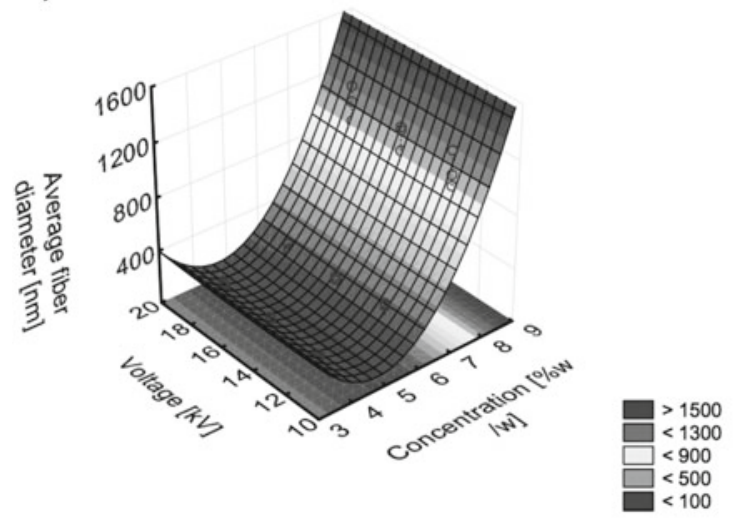

b)

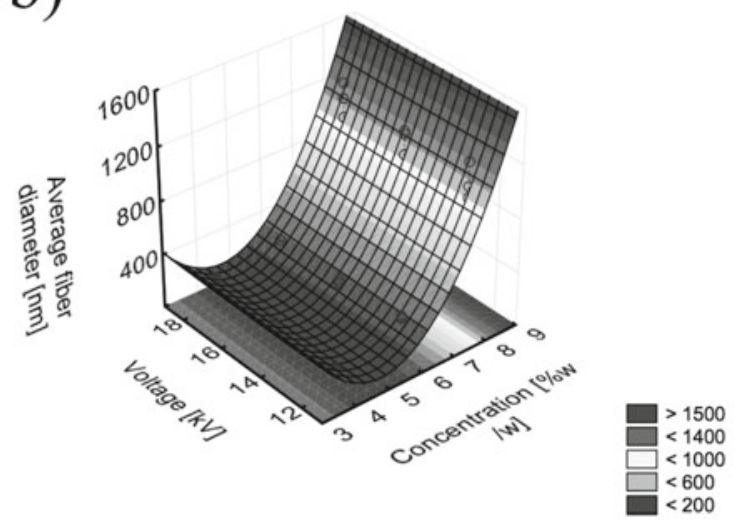

c)

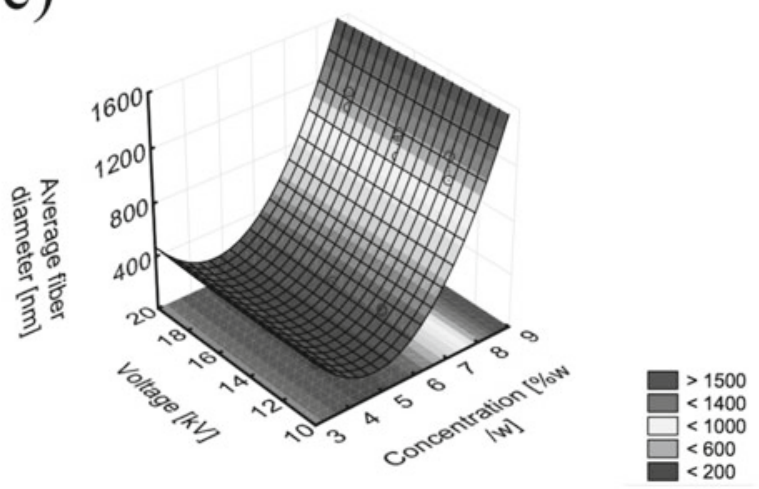

Figure 4. Best-fitted surfaces of average fiber diameter as a function of polymer concentration and voltage at a) $1 \mathrm{ml} \cdot \mathrm{h}^{-1}$, b) $\left.2 \mathrm{ml} \cdot \mathrm{h}^{-1}, \mathrm{c}\right) 3 \mathrm{ml} \cdot \mathrm{h}^{-1}$ solution feed rate for electrospinning 
Other investigated parameters, based on Pareto chart, have no influence on quality factor of resulting non-woven nanofibrous material produced from PLLA. However, for solution blow spinning, it is important to keep solution feed rate at a suitable level to avoid jet interruptions. Moreover, the proper value of an air pressure in the process of fiber formation might affect product morphology and generates more aligned fibers in the mat. On the other hand, electrospinning process depends more on solution feed rate factor in the case of maintaining stable electrospun jet. The value of applied high voltage has no influence on the quality factor, however high values of voltage might cause problems with polymer solution stability. The abovementioned lack of influence of processing parameters, other than solution concentration, on fibers diameter is stated based on analysis of simultaneous changes of those parameters. Since literature states that all processing parameters have an influence on ES and SBS process ${ }^{16,25}$, however it is caused by analysis carried out with fixed values of all parameters other than the investigated one.

\section{Morphology}

Preparation of proper scaffold for tissue engineering, drug carrier for drug delivery systems or support for wound dressing requires certain conditions to be met. One of the most important factors for most of biomedical applications of biomaterials is biocompatibility, however in many cases the morphology of applied biomaterial also might play the crucial role. The electrospinning process allows tailoring morphology of resulting nanofibrous material in terms of porosity and alignment of the fibers. In order to replace electrospinning by a more efficient way of producing nanofibrous materials for biomedical purposes, it is necessary to meet same expectations within material properties. Investigation of morphology of nanofibers from electrospinning and solution blow spinning was conducted (Fig. 6). However, it is impossible to fully compare nanofibrous mats obtained from both techniques due to differences in polymer solutions preparation process and limited possibilities of environment control.

Electrospun nanofibrous material poses highly uniform, randomly oriented fibers. Defects of the structure of nanofibrous material produced by ES did not occurred. On the other hand, solution blow spun material exhibit similar structure to those of electrospun material, however entanglement of fibers occurs. Also, an existence of lumps of polymeric material makes the non-woven mat form SBS less attractive. Since the solutions of PLLA for both processes were prepared in the different manner with different solvents, it is impossible to compare the morphology of the products form ES and SBS. However, both techniques allow tailoring of product properties and give an opportunity to produce nanofibrous materials from biodegradable and biocompatible polymer - PLLA.

Comparing the size of single fibers and average fibers diameter for materials resulting from both methods, solution blow spun fiber entanglement is negligible, because of the size of single fiber resulting from this
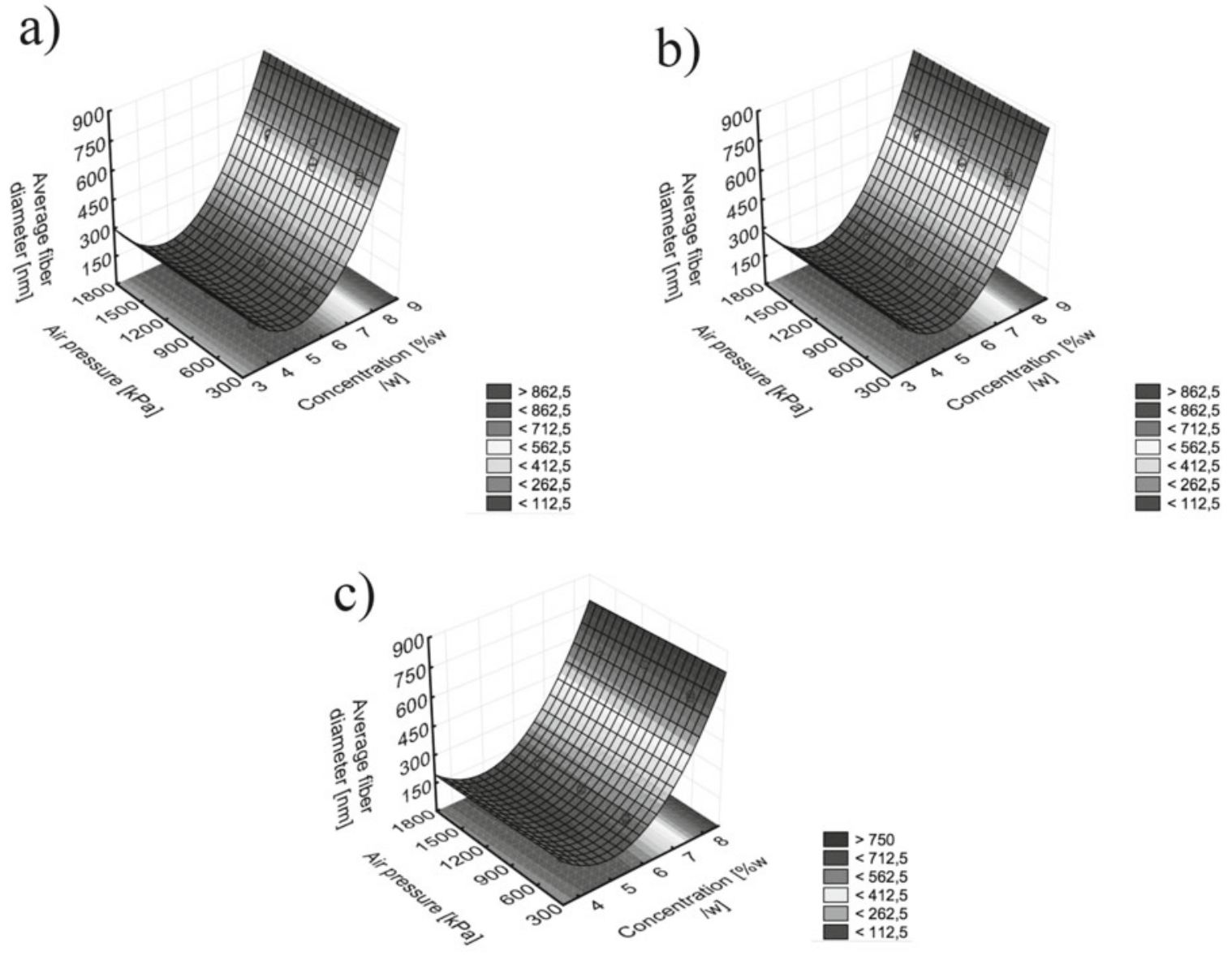

Figure 5. Best-fitted surfaces of average fiber diameter as a function of polymer concentration and air pressure at a) $15 \mathrm{ml} \cdot \mathrm{h}^{-1}$, b) $\left.30 \mathrm{ml} \cdot \mathrm{h}^{-1}, \mathrm{c}\right) 45 \mathrm{ml} \cdot \mathrm{h}^{-1}$ solution feed rate for solution blow spinning 
method. Solution blow spinning allows obtaining nanofibrous materials with average diameter of fibers between about $131 \pm 12 \mathrm{~nm}$ and about $678 \pm 56 \mathrm{~nm}$ with narrow diameter distribution. Electrospinning process allows obtaining nanofibrous materials with average diameter of fibers between about $146 \pm 14 \mathrm{~nm}$ and about $1259 \pm 35$ $\mathrm{nm}$. In the case of the thickest nanofibers obtained in both methods, solution blow spinning offers possibility of producing fibers closed to nanometric region, rather than electrospinning.

\section{Preliminary biocompatibility evaluation}

It is well established in the previously published research, that electrospun mats are one of the most promising and the closest to commonly applied biomaterials in areas like tissue engineering and three-dimensional cell cultures $^{\text {26-29. }}$. Cultures of CP5 chondrocytes were performed on nanofibrous scaffolds produced by electrospinning and solution blow spinning in order to compare the level of biocompatibility of investigated materials. Samples of the materials with cells after one day and seven days of cultures were fixed and gold-coated for SEM analysis. The biocompatibility of the materials was evaluated based on the morphology of cells aggregated on the surface on
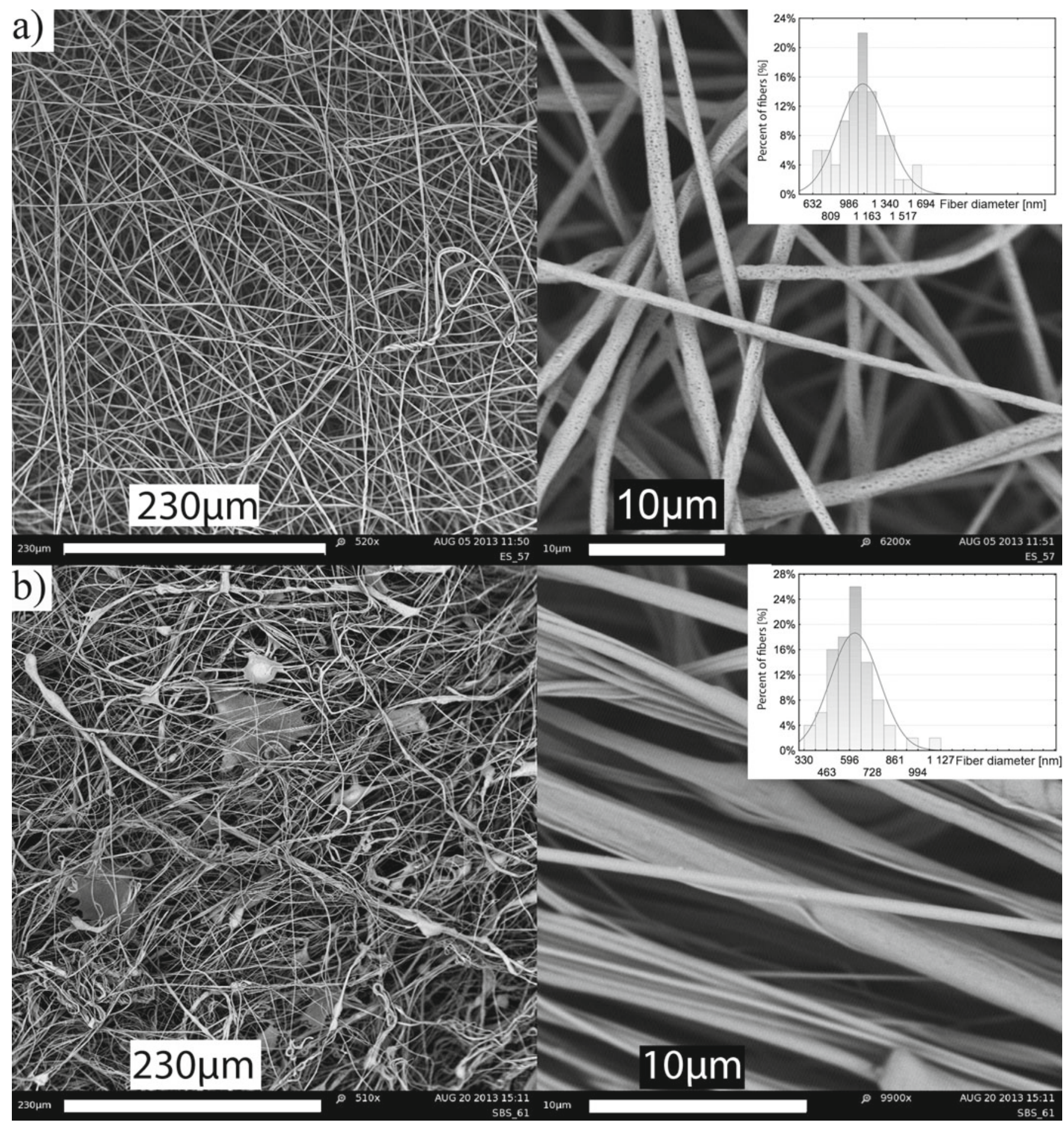

Figure 6. SEM micrographs: a) electrospun nanofibers; b) solution blow spun nanofibers. Electrospinning conditions: concentration $-8 \% \mathrm{w} / \mathrm{w}$, voltage $-18 \mathrm{kV}$, solution feed rate $-2 \mathrm{~mL} \cdot \mathrm{h}^{-1}$, temperature: $23^{\circ} \mathrm{C}$, relative humidity $-42 \%$. Solution blow spinning conditions: concentration $-8 \% \mathrm{w} / \mathrm{w}$, pressure $-1.5 \mathrm{kPa}$, solution feed rate $-15 \mathrm{~mL} \cdot \mathrm{h}^{-1}$, temperature: $23^{\circ} \mathrm{C}$, relative humidity $-43 \%$ 


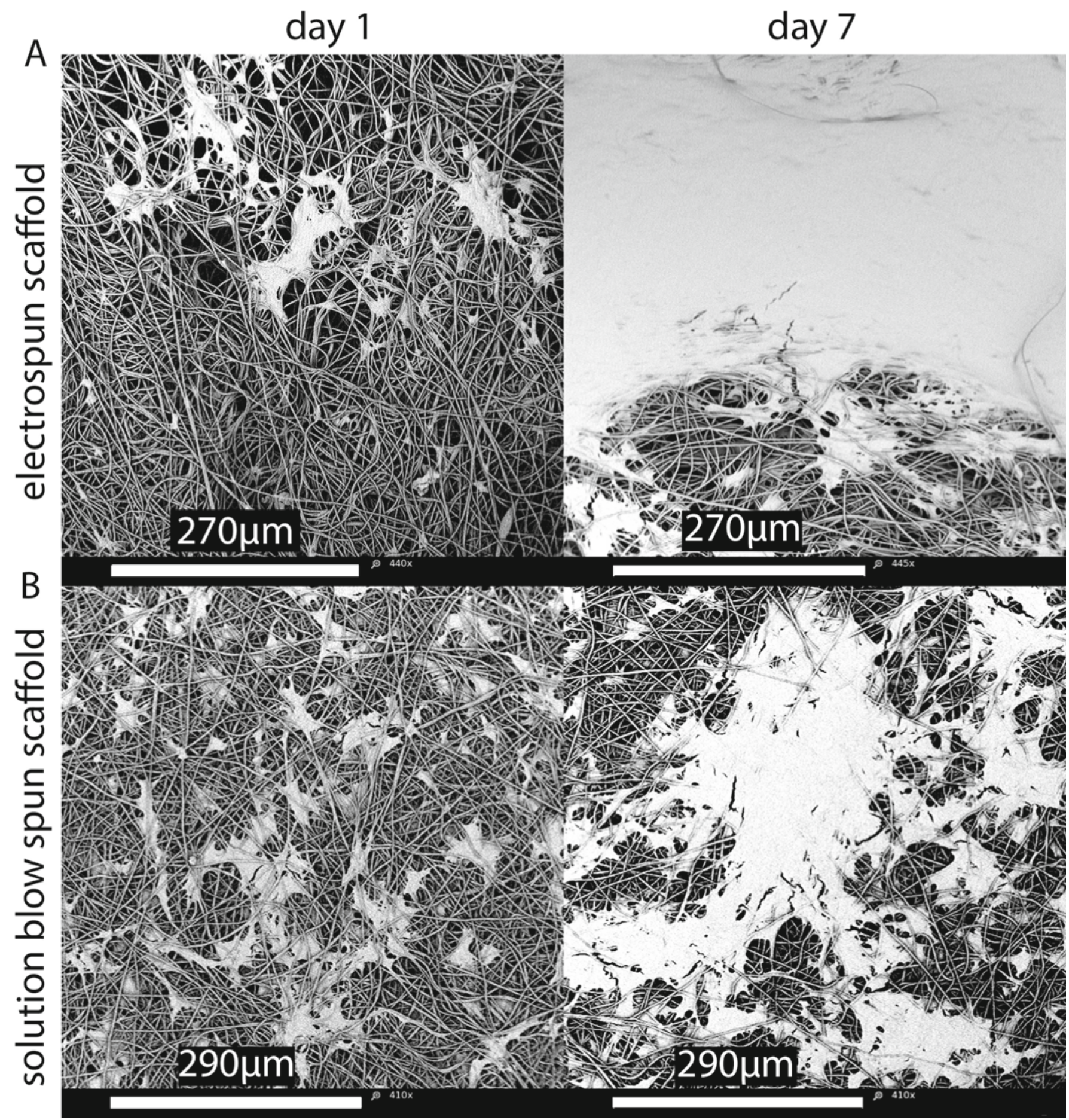

Figure 7. Morphology of aggregates of CP5 cells cultured on the electrospun (A) and solution blow spun (B) scaffolds

studied materials (Fig. 7). After seven days of culture, CP5 chondrocytes almost equally covered the surface of the materials produced by both, electro- and solution blow spinning methods. Based on SEM micrographs the biocompatibility of PLLA nanofibrous material obtained from solution blow spinning is similar to these obtained from electrospinning process.

\section{CONCLUSION}

Growing interest in the area of biomedical engineering, especially in the field of tissue engineering, generates need for production of designed fully biocompatible scaffolds for human cell culture. Electrospinning, as a method for producing scaffolds for tissue engineering, is well known since more than thirty years, but still has not been developed enough to be applied in industrial scale.
Solution blow spinning, a new technique for nanofibers production was suggested as a solution to scaling-up the nanofibers manufacturing process to the commercial level.

Since electrospinning gave the possibilities of tailoring product properties, solution blow spinning needs to met the same conditions as electrospinning. In order to evaluate possibility of replacing electrospinning by solution blow spinning the comparative studies were conducted. The polymer concentration in the spinning solution was found as a key parameter for ES and SBS process having influence on nanofiber diameter and morphology of nanofibrous mat. Properties like average fiber diameter and morphology of nanofibrous sheets produced from SBS might be adjusted, as well as properties of electrospun nanofibrous sheets. Moreover, preliminary 
biocompatibility test suggests that materials from both methods might be suitable for any biomedical application.

\section{ACKNOWLEDGMENT}

Presented work was supported by the research grant of European Committee "EraNet - ArtiCart, Artificial Cartilage".

\section{Authors' contributions}

MW carried out most of the experiments, drafted and wrote the manuscript. MP participated in and supervised the experiments with CP5 cells. TC supervised the study, participated in its design and coordination. All authors critically read and approved the final manuscript.

\section{Competing interests}

All authors declare that they have no competing interests.

\section{LITERATURE CITED}

1. Formhals, A. (1934). U.S. Patent No. 1,975,504. United States Patent Office.

2. Ciach, T. \& Gradoń, L. (1996). Highly efficient filtering materials. J. Aerosol Sci. 27(1), S613-S614. DOI: 10.1016/00218502(96)00379-5.

3. Huang, Z.M., Zhang, Y.Z., Kotaki, M. \& Ramakrishna, S. (2003). A review on polymer nanofibers by electrospinning and their applications in nanocomposites. Compos. Sci. Technol. 63(15), 2223-2253. DOI: 10.1016/S0266-3538(03)00187-7.

4. Kim, F.S., Ren, G. \& Jenekhe, S.A. (2010). One-Dimensional Nanostructures of $\pi$-Conjugated Molecular Systems: Assembly, Properties, and Applications from Photovoltaics, Sensors and Nanophotonics to Nanoelectronics. Chem. Mater. 23(3), 682-732. DOI: $10.1021 / \mathrm{cm} 102772 x$.

5. Niu, R., Qiao, J., Yu, H., Nie, J. \& Yang, D. (2011). Electrospun composite nanofibrous membrane as wound dressing with good adhesion. Front. Chem. China 6(3), 221-226. DOI: 10.1007/s11458-011-0244-7.

6. Okuda, T., Tominaga, K. \& Kidoaki, S. (2010). Timeprogrammed dual release formulation by multilayered drugloaded nanofiber meshes. J. Control. Release. 143(2), 258-264. DOI: 10.1016/j.jconrel.2009.12.029.

7. Koh, H.S., Yong, T., Chan, C.K. \& Ramakrishna, S. (2008). Enhancement of neurite outgrowth using nano-structured scaffolds coupled with laminin. Biomaterials. 29(26), 3574-3582. DOI: 10.1016/j.biomaterials.2008.05.014.

8. Mo, X.M., Xu, C.Y., Kotaki, M. \& Ramakrishna, S. (2004). Electrospun P(LLA-CL) nanofiber: a biomimetic extracellular matrix for smooth muscle cell and endothelial cell proliferation. Biomaterials 25(10), 1883-1890. DOI: 10.1016/j. biomaterials.2003.08.042.

9. Jang, J.H., Castano, O. \& Kim, H.W. (2009). Electrospun materials as potential platforms for bone tissue engineering. Adv. Drug Deliver. Rev. 61(12), 1065-1083. DOI: 10.1016/j. addr.2009.07.008.

10. Ramakrishna, S., Fujihara, K., Teo, W.E., Lim, T.C. \& Ma, Z. (2005). An Introduction to Electrospinning and Nanofibers. World Scientific Publishing. Singapore.

11. Ravichandran, R., Sundarrajan, S., Venugopal, J.R., Kukherjee, S. \& Ramakrishna, S. (2012). Advances in Polymeric Systems for Tissue Engineering and Biomedical Applications. Macromol. Biosci. 12(3), 286-311. DOI: 10.1002/ mabi.201100325.

12. Li, D. \& Xia, Y. (2004). Electrospinning of Nanofibers: Reinventing the Wheel? Adv. Mater. 16(14), 1151-1170. DOI: 10.1002/adma.200400719.
13. Persano, L., Camposeo, A., Tekmen, C. \& Pisignano, D. (2013). Industrial Upscaling of Electrospinning and Applications of Polymer Nanofibers: A Review. Macromol. Mater. Eng. 298(5), 504-520. DOI: 10.1002/mame.201200290.

14. Angammana, C.J. \& Jayaram, S.H. (2011). The Effects of Electric Fields on the Multijet Electrospinning Process and Fiber Morphology. IEEE T. Ind. Appl. 47(2), 1028-1035. DOI: 10.1109/TIA.2010.2103392.

15. Niu, H., Wang, X. \& Lin, T. (2012). Upward Needleless Electrospinning of Nanofibers. J. Eng. Fiber Fabr. Special Issue - July, 17-22.

16. Medeiros, E.S., Glenn, G.M., Klamczynski, A.P., Orts, W.J. \& Mattoso, L.H.C. (2009). Solution Blow Spinning: A New Method to Produce Micro- and Nanofibers from Polymer Solutions. J. Appl. Polym. Sci. 113, 2322-2330. DOI: 10.1002/ app.30275.

17. Oliveira, J.E., Moraes, E.A., Costa, R.G.F., Afonso, A.S., Mattoso, L.H.C., Orts, W.J. \& Medeiros, E.S. (2011). Nano and Submicrometric Fibers of Poly(D,L-Lactide) Obtained by Solution Blow Spinning: Process and Solution Variables. J. Appl. Polym. Sci. 122(5), 3396-3405. DOI: 10.1002/app.34410.

18. Sabbatier, G., Le Nouën, D., Chevallier, P., Durand, B., Laroche, G. \& Dieval, F. (2012). Air spun poly(lactic acid) nanofiber scaffold degradation for vascular tissue engineering: A 1H NMR study. Polym. Degrad. Stabil. 97(8), 1520-1526. DOI: 10.1016/j.polymderadstab.2012.04.017.

19. Gupta, B., Revagade, N. \& Hilborn, J. (2007). Poly(lactic acid) fiber: An overview. Prog. Polym. Sci. 32(4), 455-482. DOI: 10.1016/j.progpolymsci.2007.01.005.

20. Wojasiński, M., Faliszewski, K. \& Ciach, T. (2013). Electrospinning Production of PLLA Fibrous Scaffolds for Tissue Engineering. CoMT. 4(1), 9-15.

21. Pilarek, M., Grabowska, I., Senderek, I., Wojasiński, M., Janicka, J., Janczyk-Ilach, K. \& Ciach, T. (2014). Liquid perfluorochemical-supported hybrid cell culture system fo proliferation of chondrocytes on fibrous polylactide scaffolds. Bioproc. Biosys. Eng. In press, 1-9. DOI: 10.1007/s00449-014-1143-3.

22. Bhardwaj, N., Kundu, S.C. (2010). Electrospinning: A fascinating fiber fabrication technique. Biotechnol. Adv., 28(3), 325-347. DOI: 10.1016/k.biotechadv.2010.01.004.

23. Zong, X., Kim, K., Fang, D., Ran, S., Hsiao, B.S., Chu, B. (2002). Structure and process relationship of electrospun bioabsorbable nanofiber membranes. Polymer. 43(16), 4403-4412. DOI: 10.1016/S0032-3861(02)00275-6.

24. Oliveira, J.E., Mattoso, L.H.C., Orts, W.J., Medeiros, E.S. (2013). Structural and morphological characterization of micro and nanofibers produced by electrospinning and solution blow spinning: A comparative study. Adv. Mater. Sci. Eng. Article number 409572. DOI: 10.1155/2013/409572.

25. Deitzel, J.M., Kleinmeyer, J., Harris, D., Beck Tan, N.C. (2001). The effect of processing variables on the morphology of electrospun nanofibers and textiles. Polymer. 42(1), 261-272.

26. Lannutti, J., Reneker, D., Ma, T., Tomasko, D. \& Farson, D. (2007). Electrospinning for tissue engineering scaffolds. Mat. Sci. Eng. C, 27(3), 504-509. DOI: 10.1016/j.mscc.2006.05.019.

27. Beachley, V. \& Wen, X. (2010). Polymer nanofibrous structures: Fabrication, biofunctionalization, and cell interactions. Prog. Polym. Sci. 35(7), 868-892. DOI: 10.1016/j. progpolymsci.2010.03.003.

28. Song, X., Ling, F., Ma, L., Yang, C. \& Chen, X. (2013). Electrospun hydroxyapatite grafted poly(L-lactide)/poly(lactic-co-glycolic acid) nanofibers for guided bone regeneration membrane. Compos. Sci. Technol. 79, 8-14. DOI: 10.1016/j. compscitech.2013.02.014.

29. Tucker, N., Stanger, J.J., Staiger, M.P., Razzaq, H. \& Hoffman, K. (2012). The History of the Science and Technology of Electrospinning from 1600 to 1995. J. Eng. Fiber Fabr., Special Issue - July, 63-73. 